\title{
The health perceptions, dengue knowledge and control willingness among Dai ethnic minority in Yunnan Province, China
}

\author{
Hui Liu ${ }^{1,2}$, Cheng-Jiang Fang ${ }^{3}$ and Jian-Wei $X u^{1,2^{*}}$
}

\begin{abstract}
Background: Outbreaks of dengue fever are often found among Dai ethnical communities along China-Myanmar border. The objective of this study was: 1) to investigate residents' health perceptions, knowledge and control willingness to participate in dengue control and 2) to identify factors associated with control willingness among the Dai ethnic community.

Methods: This is a mixed method study of a cross-sectional design, in which qualitative in-depth interviews and quantitative household questionnaire surveys are included.

Results: Questionnaire was administered to 261 household heads, and in-depth interview was conducted with 18 key informants. Of them, many participants (70\%, 182/259) and 12 key informants (66.7\%) from the two rural communities believed that the Lord Buddha would protect the good people. Majority of the participants (81.4\%, 206/253) knew that fever was one of dengue fever symptoms and most of them $(82.2 \%, 213 / 259)$ indicated that mosquitoes could transmit dengue fever. However, only one third of the participants $(30.1 \%, 78 / 259)$ indicated the perceived susceptibility of dengue fever, and only a half of them $(50.2 \%, 130 / 259)$ indicated the perceived severity of dengue fever. Multivariate logistic analysis (MLA) indicated that the participants with family wealth index (FWI) 4-5 (OR: 22.9728; 95\%Cl: 2.4257-217.5688, $p=0.0063$ ) were more likely to turn containers upside down (TCUD) compared to those with FWI 1-3; and the participants in the urban community (OR: 0.0239; 95\%Cl: 0.0019-0.3032, $p=0.004)$ were less likely to TCUD compared to those in the two rural communities. Around one third of the participants $(36.8 \%, 96 / 239)$ reported that they were willing to seek treatment first for dengue fever from public health facilities. The MLA identified that the participants with the perceived severity of dengue fever (OR: 5.0564; 95\%Cl: 2.0672-12.3683, $p=0.0004$ ), and with beliefs of sound hygiene helpful to people's health (OR: 11.5671; $95 \%$ Cl: $2.0505-65.2502, p=0.0055)$ were more likely to seek treatment first for dengue fever from the public health facilities.
\end{abstract}

\footnotetext{
* Correspondence: xjw426@163.com

${ }^{1}$ Yunnan Institute of Parasitic Diseases, Yunnan Provincial Key Laboratory of

Vector-borne Diseases Control and Research, Xiyuan Road, Pu'er City 665000,

China

${ }^{2}$ Institute of Pathogens and Vectors, Basic Medical College, Dali University,

Wanhua Road, Xiaguang District, Dali City 671000, China

Full list of author information is available at the end of the article
}

(c) The Author(s). 2021 Open Access This article is licensed under a Creative Commons Attribution 4.0 International License, which permits use, sharing, adaptation, distribution and reproduction in any medium or format, as long as you give appropriate credit to the original author(s) and the source, provide a link to the Creative Commons licence, and indicate if changes were made. The images or other third party material in this article are included in the article's Creative Commons licence, unless indicated otherwise in a credit line to the material. If material is not included in the article's Creative Commons licence and your intended use is not permitted by statutory regulation or exceeds the permitted use, you will need to obtain permission directly from the copyright holder. To view a copy of this licence, visit http://creativecommons.org/licenses/by/4.0/ The Creative Commons Public Domain Dedication waiver (http://creativecommons.org/publicdomain/zero/1.0/) applies to the data made available in this article, unless otherwise stated in a credit line to the data. 
Conclusion: The study finds that most of Dai people have sound knowledge. However, health educational interventions should target to promote the perceived susceptibility and the perceived severity of dengue fever among Dai people.

Keywords: Health perception, Dengue fever, Knowledge, Willingness, Dai ethnic minority, China

\section{Background}

Dengue fever is one of the hardest-to-control arboviral diseases in the world. The World Health Organization (WHO) estimated 284-528 million dengue infections yearly in 128 territories [1,2]. Dengue also causes a substantial economic burden to governments and households [2, 3]. In China, dengue fever incidence and areas affected have steadily increased since $2000[4,5]$. Dengue has become one of the main public health problems, while malaria was successfully controlled along ChinaMyanmar border [6, 7]. The Dai people in Yunnan Province, Southwestern China, are one of the most affected populations by dengue fever outbreaks in China [8].

There are no effective antiviral therapies currently available for dengue fever and to date the only licensed dengue vaccine is not fully protectable [9]. Thus intensified vector control is one of the most widely used strategies to prevent dengue virus transmission [10,11]. Water containers and discarded tires left by local people are primary productive habitats of Aedes $s p$ mosquitoes. Clearance of the habitats can reduce density of Aedes sp mosquitoes and further to reduce dengue virus transmission [10]. Community involvement is one of effective strategies to manage the habitats of Aedes $s p$ mosquitoes [11, 12]. Early diagnosis is very important for effectively clinical management to improve patient prognosis and to reduce transmission of dengue virus [13, 14]. In China, laboratory test for dengue fever is only available in public health facilities, thus that suspected dengue fever patients seek appropriate treatment first from public health facilities is helpful to detection of dengue fever [6].

Local people's health beliefs and knowledge of dengue fever may likely influence their willingness to participate in dengue fever control and then further influence transmission of dengue virus $[14,15]$. The health beliefs are generally shaped by people's expectancies about the environmental cues that are about how events are connected - about what leads to what, the consequences of their own actions that are about how individual behaviors are likely to influence outcomes, and their own competence to perform the behavior needed to influence outcomes. The basic concepts of the Health Belief Model (HBM) are health knowledge, health concerns (perceived susceptibility and perceived severity), perceived cost-benefits, perceived barriers to taking health action, cue to action and self-efficacy [16-18]. Chinese Government has put a highly political will and commitment to dengue control, and also increased investment in researches on biomedicine, surveillance system and public responses since the epidemic of dengue fever in Guangdong Province [12]. Nevertheless, the HBM study has rarely been applied in an attempt to understand social determinants of dengue control among the ethnic minorities in China.

This study was carried out among three Dai ethnic communities from December 2017 to April 2018, prior to the local dengue transmission season. The objective of this study was: 1) to investigate residents' health beliefs, knowledge and willingness to participate in dengue fever control and 2) to identify factors associated with the control willingness among the Dai ethnic community.

\section{Methods}

\section{Study design and setting}

This is a mixed method study of a cross-sectional design, in which qualitative in-depth interviews and quantitative household questionnaire surveys were included. For this study, three villages with high, middle and low incidences of dengue fever in 2017 were sampled from Xishuangbanna Prefecture, Yunnan Province (Fig. 1). A total of 18 key informants (six per village) were sampled for qualitative in-depth interviews. Based on an estimated $20 \%$ of adult people who had knowledge that dengue virus is transmitted by mosquitoes, standard value normal distribution at 95\% confidence levels and 5\% precision, a sample size of at least 250 household heads for quantitative household questionnaire survey was determined [19]. Assuming a population with higher incidence of a disease may be more knowledgable about the disease, the crude sampling ratio was set up around 1:2:2 for the three communities experienced high, middle and low incidences of dengue fever in 2017.

Xishuangbanna Prefecture has three counties (i.e., Menghai, Jinghong and Mengla) with a total population of 1.2 million, including 350 thousand of Dai people. Dai people, one of the main ethnicities in the Greater Mekong Subregion, are also known as Shan in Myanmar, Thai Yai in Thailand and Lao in Lao PDR. All the participants of this study were Dai people from the three communities with high, middle and low incidences of dengue fever in 2017. Manduo is an urbanized 


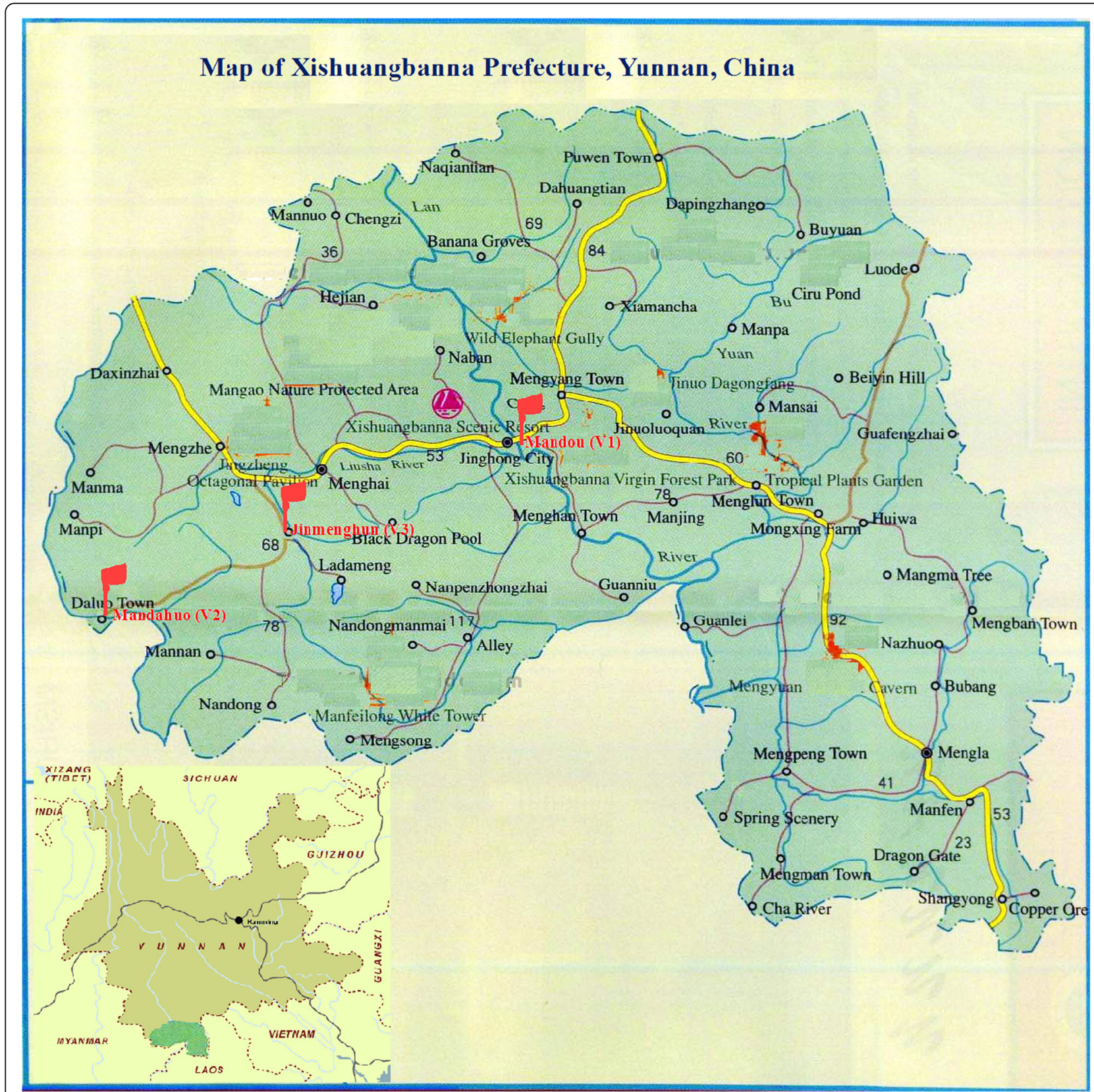

Fig. 1 Location of study site: The red flags indicate the study site in Xishuangbanna Prefecture, Yunnan, China. The figure was generated by using the drawing tool of Microsoft Windows 10 software based on original Yunnan map and Xishuangbanna map collected in the Map Bank of Yunnan Institute of Parasitic Diseases (YIPD). The bank is open access and use to YIPD's staff without any charges or barriers

community with a population of 889 and with the high incidence of dengue fever in 2017 on the north bank of the Lancangjiang River (the upper section of Mekong River) in Jinghong City. Mandahuo is a village with a population of 675 and with the middle incidence of dengue fever in 2017 on the China-Myanmar border, Daluo Town, Menghai County. Jingmenghun is a rural community with a population of 879 and with only one imported dengue fever case in 2017 in Menghun town, Menghai County (Fig. 1). Manduo and Mandahuo are suitable for reproduction of mosquitoes and transmission of vector-borne diseases due to their lower attitude (around $600 \mathrm{~m}$ ), tropical climate and adequate precipitation. The key informants estimated that about $20 \%$ of people in Mandou had dengue fever episodes, and 1.5\% of people in Mandahuo had dengue fever episodes in 2017, respectively. Jingmenghun is less appropriate for reproduction of mosquitoes and transmission of vectorborne diseases because of its altitude of $1198 \mathrm{~m}$ and cool climate. 


\section{In-depth interviews}

The qualitative in-depth interview guideline (Additional file 1) covered: 1) local health problems, people's beliefs related to religions, causes of diseases and perceived local dengue situation; 2) dengue knowledge consisted of symptoms of dengue fever, morphological characteristics of Aedes spp, breeding sites and biting time of Aedes spp; and 3) willingness to participate dengue control, i.e. management of Aedes spp breeding sites and seeking treatment first for dengue fever from public health facilities. The qualitative in-depth interviews were conducted with six key informants at each community (a total of 18 key informants, three communities), including one health worker, three village leaders and two community representatives who were selected by the other villagers. All of the key informants can understand and speak mandarin. Two investigators worked together to conduct every interview, one investigator discussed with the interviewees, and another took notes in Chinese.

\section{Household questionnaire survey}

A household was a sampling unit that was defined as all people eating from the same cooking pot. The quantitative household questionnaire (Additional file 2) consisted of socio-demographic characteristics, health beliefs, dengue fever knowledge, willingness to participate dengue control and household's physical assets. Where the health beliefs consisted of nine items, the dengue fever knowledge consisted of 26 items and the willingness consisted of 13 items. The household's physical assets, including housing, walls, roofs, transportation tools and family belongings, were used to categorize family wealth index (FWI) into five levels (Table 1). The simple random sampling was used to select households based on existing household numbers from local authorities for this study. All participants can understand and speak Mandarin, thus the surveys were conducted in Mandarin. Investigators first visited each sampled household and introduced to the household head about this survey's purpose and answered questions raised. Following an oral informed consent obtained, the questionnaire was administered to the household head.

\section{Statistical analysis}

Both qualitative and quantitative data were entered with the Microsoft Excel 2007. The transcriptions of qualitative in-depth interviews were coded according to topics and then entered into cells in Microsoft Office Excel 2007. The same content records were combined by code sequencing. Two researchers generated themes independently, and then they compared and discussed their findings to synthesize findings under the same spectrums to finalize the health themes $[8,14]$. The health themes identified from the qualitative study were independently presented first closely following the respondents' characteristics. To synthesize the information of qualitative study with the results of quantitative data analysis well, the more detailed results of qualitative study are interspersed with the quantitative data in each part of the results.

All statistical analyses of the quantitative data were performed with the Epi Info 7.2 (Centers for Disease Control and Prevention, USA). Non-response and irrelevant answers were treated as missing values and therefore excluded from the analyses. The proportion and its 95\% confidence interval (CI) were calculated for each item of health beliefs, knowledge, prevention and treatment-seeking willingness. The proportions of each item were compared by chi-squared Fisher's exact test across the three study sites. Values of $p<0.05$ were considered statistically significant.

A multivariate logistic analysis (MLA) was used to identify factors associated with control willingness. The outcome " 1 " in the MLA model means that a household's willingness of turning containers upside down, and seeking treatment first from public facilities if one of the family members had suspected symptoms of dengue fever. In modeling strategy, the independent variables of univariate logistic analysis were items with values of $P<0.05$ in the chi-squared Fisher's exact test across the three study sites. In addition to this criterion, two counties (Jinghong and Menghai) where the three study sites are located were included in the univariate logistic analysis in considering the difference of the dengue fever incidence and the urbanization degree of the two counties. The independent variables were included in

Table 1 Principal components for construction of the family wealth index (FWI)

\begin{tabular}{lll}
\hline Family wealth index & Housing characteristics & Transportation tools \\
\hline $\mathbf{1}$ Most poor & Bamboo walls and sheet iron roofs & None \\
$\mathbf{2}$ Mid low & Wood walls and sheet iron roofs & Bicycles \\
$\mathbf{3}$ Middle & Brick walls, wood girders and terracotta roofs & Motorcycles \\
$\mathbf{4}$ Mid high & Brick concrete walls and terracotta roofs & Tractors \\
$\mathbf{5}$ Least poor & Steel and concrete & Cars \\
\hline
\end{tabular}


the MLA model if they had a value of $p<0.25$ on the univariate logistic analysis.

\section{Concept definition}

Local people's health beliefs can influence their knowledge and control willingness. Health belief here was defined as the general perception of elements relevant to health and disease and not limited to unique beliefs about dengue fever. It shows local perception of the effect of socioeconomic, natural and religious elements on their health and their perception of disease causes. In addition, respondent's knowledge regarding dengue includes their knowledge of clinical symptoms, vectors, prevention, treatment and perceived severity of dengue fever [8]. Control willingness is defined as a participant's attitude about participating in community-based vector control like cleaning dumps and turn containers upside down, and seeking treatment first from public health facilities if one of the family members had suspected symptoms of dengue fever [14].

\section{Results}

Participants' characteristics

A total of 18 key informants with age range 30 to 57 years old were interviewed in depth. There were more males than females (10 vs. 8) for the key informants. A total of 261 valid questionnaires were completed. As Table 2 shows, the mean age of the sample was 43.4 years old (median: 41.0, range: 17-84), and the mean age of the participants in the urban community $(39.2$ years) was younger compared to those in the two rural communities (45.8 years in Mandahuo and 43.6 years in Jingmenhun). There were fewer males $(36.8 \%, 96 / 261)$ than females among participants of the quantitative study. About a half of participants $(49.4 \%, 129 / 261)$ had formal school education for less than 6 years. Most of the participants $(98.8 \%, 256 / 259)$ of the quantitative study and the key informants $(94.4 \%, 17 / 18)$ of the qualitative study were Buddhists. Most of participating households $(95 \%, 246 / 259)$ were not poor with a family wealth index (FWI) 4-5.

\section{Health themes identified from qualitative study}

All participants of the qualitative study were Dai people. The analysis of qualitative data identified five health themes: 1) Differences of key informant's beliefs existed between the urbanized community (Manduo) and the two rural communities (Mandahuo and Jingmenghun). All key informants believed keeping living and working areas clean to benefit for their health. However, the six key informants from the urbanized community did not believe that the Lord Buddha could bless and protect good people from diseases despite that five of them were Buddhist. In contrast, the 12 key informants of the two rural communities believed the Lord Buddha could protect them from diseases if they performed well. The six key informants from the urbanized community denied any connections between diseases and economic conditions, but they believed that human health was associated with natural environment. The 12 key informants of the two rural communities believed that both economic conditions and natural environment were associated with their health. 2) Key informants believed that most of their villagers had sound knowledge on the symptoms, vector and transmission of dengue fever. The key informants in the two communities with locally infected dengue fever cases (Manduo and Mandahuo) knew more about dengue fever than the key informants in the community with only one imported dengue case (Jingmenghun) in 2017. Their distinct knowledge gap between two kinds of the communities with locally infected dengue fever cases and the community with only one imported dengue case was about Aedes spp and Aedes larvae breeding sites, clinical symptoms, causes of dengue fever and dengue prevention. 3) Most of the key informants perceived dengue fever as a severe disease that could take away people's lives. 4) Key informants perceived that the governmental requirement of social mobilization worked to clear the habitat of Aedes sp larva. 5) Most of the key informants perceived that Dai people were willing to seek treatment first from public health facilities if they recognized that they were infected with dengue virus.

\section{Health perceptions in general}

As shown in Table 2, most of the participants $(70.0 \%$, 182/260) believed that the Lord Buddha would protect good people. A significantly lower proportion of the participants $(5 \%, 3 / 60)$ in the urbanized community had the belief that the Lord Buddha would protect good people compared to the participants in the two rural communities (100\%, 99/99 in Mandahu and 80\%, 80/100 in Jingmenghun). During in-depth interview in Mandahuo, one key informant noted "The Buddha will bless and protect good people. If anyone does good things, he or she will get the blessings from the Lord Buddha". However, only one of the key informants agreed this belief in the urbanized community.

Most of the participants $(76.5 \%, 198 / 259)$ agreed that human health were associated with natural factors. A significantly higher proportion of the participants in the urbanized community $(100 \%, 59 / 59)$ believed that people's diseases were related to the poor hygiene compared to those in the two rural communities $(80.8 \%, 80 / 99$ in Mandahuo and 81\%, 81/100 in Jingmenghun). During in-depth interview in the urbanized community, one key informant noted "The hotter weather, the more people get ill. There are fewer diseases in cold weather days"; 
Table 2 Demographic characteristics, health perceptions, dengue knowledge and control willingness $(N=261)$

\begin{tabular}{|c|c|c|c|c|c|}
\hline Variables & $\begin{array}{l}\text { Total No. (\%, } \\
\left.95 \mathrm{Cl}^{\mathrm{a}}\right), n=261\end{array}$ & $\begin{array}{l}\text { No. }(\%, 95 \mathrm{Cl}) \text { in } \\
\text { Manduo, } n=61\end{array}$ & $\begin{array}{l}\text { No. }\left(\%, 95 \mathrm{Cl}^{\mathrm{a}}\right) \text { in } \\
\text { Mandahuo, } n=100\end{array}$ & $\begin{array}{l}\text { No. }\left(\left[\%, 95 \mathrm{Cl}^{\mathrm{a}}\right) \text { in }\right. \\
\text { Jingmenghun, } n=100\end{array}$ & $P$-value \\
\hline \multicolumn{6}{|l|}{ Demographics } \\
\hline Male sex of household heads & $\begin{array}{l}96(36.8,30.9- \\
43.0)\end{array}$ & $25(41.0,28.6-54.3)$ & $27(27.0,18.6-36.8)$ & $44(44.0,34.1-54.3)$ & 0.0331 \\
\hline \multicolumn{6}{|l|}{ Age of household heads (years) } \\
\hline $18-40$ & $\begin{array}{l}124(47.5,41.3- \\
53.8)\end{array}$ & $39(63.9,50.6-75.8)$ & $42(42.0,32.2-52.3)$ & $43(43.0,33.1-53.3)$ & 0.0134 \\
\hline $41-84$ & $\begin{array}{l}137(52.5,46.2- \\
58.7)\end{array}$ & $22(36.1,24.2-49.4)$ & $58(58.0,47.7-67.8)$ & $57(57.0,46.7-66.9)$ & 0.0134 \\
\hline School education (years) & 43 & & & & \\
\hline$\leq 6$ & $\begin{array}{l}129(49.4,87.2- \\
55.7)\end{array}$ & $13(21.3,11.9-33.7)$ & $36(36.0,26.6-46.2)$ & $80(80.0,70.8-87.3)$ & $<0.0001$ \\
\hline $7-9$ & $\begin{array}{l}58(22.2,17.3- \\
27.8)\end{array}$ & $30(49.2,36.1-62.3)$ & $12(12.0,6.4-20.0)$ & $16(16.0,9.4-24.7)$ & $<0.0001$ \\
\hline$>9$ & $\begin{array}{l}74(28.4,23.0- \\
34.2)\end{array}$ & $18(29.5,18.5-42.6)$ & 52(52. 0, 41.8-62.1) & $4(4.0,1.1 .9-9.9)$ & $<0.0001$ \\
\hline Family wealth index & $n=259$ & $n=59$ & $n=100$ & $n=100$ & \\
\hline 1 Most poor & $10(3.9,0-7.0)$ & $1(1.7,0-9.1)$ & $8(8.0,3.5-15.2)$ & $1(1.0,0-5.5)$ & 0.0220 \\
\hline 2 Mid low & $0(0,0-1.4)$ & $0(0,0-0.6)$ & $0(0,0-3.6)$ & $0(0,0-3.6)$ & - \\
\hline 3 Middle & $3(1.2,0.2-3.4)$ & $2(3.4,0.4-11.7)$ & $1(1.0,0-5.5)$ & $0(0,0-3.6)$ & - \\
\hline 4 Mid high & $\begin{array}{l}82(31.7,26.0- \\
37.7)\end{array}$ & $7(11.9,4.9-22.9)$ & $74(74.0,64.3-82.3)$ & $1(1.0,0-5.5)$ & $<0.0001$ \\
\hline 5 Least poor & $\begin{array}{l}164(63.3,57.1- \\
69.2)\end{array}$ & $49(83.3,71.0-91.6)$ & $17(17.0,10.2-25.8)$ & 98(98.0, 93.0-99.7) & $<0.0001$ \\
\hline \multicolumn{6}{|l|}{ Health beliefs in general } \\
\hline \multirow[t]{2}{*}{ Poverty is a cause of ill health } & $n=259$ & $n=60$ & $n=99$ & $n=100$ & \\
\hline & $\begin{array}{l}77(29.7,24.2- \\
35.7)\end{array}$ & $7(11.7,4.8-22.6)$ & $16(16.2,9.5-24.9)$ & $54(54.0,43.7-64.0)$ & $<0.0001$ \\
\hline \multirow{2}{*}{$\begin{array}{l}\text { People with evil practices may be } \\
\text { punished by diseases }\end{array}$} & $n=260$ & $n=60$ & $n=100$ & $n=100$ & \\
\hline & $\begin{array}{l}95(36.5,30.7- \\
42.7)\end{array}$ & $3(5.0,1.0-13.9)$ & $16(16.0,9.4-24.7)$ & $76(76.0,66.4-84.0)$ & $<0.0001$ \\
\hline $\begin{array}{l}\text { The Lord Buddha will protect good } \\
\text { people }\end{array}$ & $\begin{array}{l}182(70.0,64.0- \\
75.5)\end{array}$ & $3(5.0,1.0-13.9)$ & $99(99.0,94.6-100.0)$ & $80(80.0,70.8-87.3)$ & $<0.0001$ \\
\hline \multirow[t]{2}{*}{ All natural factors influence health } & $n=259$ & $n=60$ & $n=100$ & $n=99$ & \\
\hline & $\begin{array}{l}198(76.5,70.8- \\
81.5)\end{array}$ & $59(98.3,91.1-100.0)$ & $40(40.0,30.3-50.3)$ & $99(100.0,100.0-100.0)$ & $<0.0001$ \\
\hline $\begin{array}{l}\text { Beliefs on environments associated with } \\
\text { diseases }\end{array}$ & $n=258$ & $n=59$ & $n=99$ & $n=100$ & \\
\hline Too hot & $\begin{array}{l}120(46.5,40.3- \\
52.8)\end{array}$ & $14(23.7,13.6-36.6)$ & $9(9.1,4.2-16.6)$ & $97(97.0,91.5-99.4)$ & $<0.0001$ \\
\hline Too cold & $\begin{array}{l}186(72.1,66.2- \\
77.5)\end{array}$ & $33(55.9,42.4-68.8)$ & $68(68.7,58.6-77.6)$ & $85(85.0,76.5-91.4)$ & 0.0003 \\
\hline Too rainy & $\begin{array}{l}119(46.2,39.9- \\
52.4)\end{array}$ & $5(8.5,2.8-18.7)$ & $36(36.4,26.9-46.6)$ & $78(78.0,68.6-85.7)$ & $<0.0001$ \\
\hline Too forested & $22(8.5,5.4-12.6)$ & $0(0,0-6.1)$ & $0(0,0-3.6)$ & $22(22.0,14.3-31.4)$ & - \\
\hline Rivers, streams and clear water pools & $29(11.2,7.7-15.7)$ & $4(6.8,1.9-16.5)$ & $0(0,0-3.6)$ & $25(25.0,16.9-34.6)$ & - \\
\hline Polluted water & $\begin{array}{l}74(28.7,23.2- \\
34.6)\end{array}$ & $25(42.4,29.6-55.9)$ & $4(4.0,1.1-10.0)$ & $45(45.0,35.0-55.3)$ & $<0.0001$ \\
\hline Poor hygiene & $\begin{array}{l}220(85.3,80.3- \\
89.4)\end{array}$ & $59(100,93.9-100.0)$ & $80(80.8,71.7-88.0)$ & $81(81.0,71.9-88.2)$ & $<0.0001$ \\
\hline Beliefs on environment benefiting health & $n=260$ & $n=60$ & $n=100$ & $n=100$ & \\
\hline
\end{tabular}


Table 2 Demographic characteristics, health perceptions, dengue knowledge and control willingness ( $N=261)(C o n t i n u e d)$

\begin{tabular}{|c|c|c|c|c|c|}
\hline Variables & $\begin{array}{l}\text { Total No. (\%, } \\
\left.95 \mathrm{Cl}^{\mathrm{a}}\right), n=261\end{array}$ & $\begin{array}{l}\text { No. }(\%, 95 \mathrm{Cl}) \text { in } \\
\text { Manduo, } n=61\end{array}$ & $\begin{array}{l}\text { No. }\left(\%, 95 \mathrm{Cl}^{\mathrm{a}}\right) \text { in } \\
\text { Mandahuo, } n=100\end{array}$ & $\begin{array}{l}\text { No. }\left(\left[\%, 95 \mathrm{Cl}^{\mathrm{a}}\right) \text { in }\right. \\
\text { Jingmenghun, } n=100\end{array}$ & $P$-value \\
\hline Clean and sound hygiene & $\begin{array}{l}213(81.9,76.7- \\
86.4)\end{array}$ & $34(56.7,43.2-69.4)$ & $79(79.0,69.7-86.6)$ & $100(100.0,100.0-100.0)$ & $<0.0001$ \\
\hline No polluted water & $\begin{array}{l}100(38.5,32.5- \\
44.7)\end{array}$ & $16(27.7,16.1-39.6)$ & $8(8.0,3.5-15.2)$ & $76(76.0,66.4-84.0)$ & $<0.0001$ \\
\hline Many flowers, grass and trees & $\begin{array}{l}110(42.3,36.2- \\
48.6)\end{array}$ & $48(80.0,67.7-89.2)$ & $0(0,0-3.6)$ & $62(62.0,51.7-71.5)$ & $<0.0001$ \\
\hline Good hygiene can reduce diseases & $\begin{array}{l}210(80.9,75.5- \\
85.4)\end{array}$ & $25(41.7,29.1-55.1)$ & $96(96.0,90.1-98.9)$ & $89(89.0,81.2-94.4)$ & $<0.0001$ \\
\hline Perceived severity & $n=259$ & $n=60$ & $n=100$ & $n=99$ & \\
\hline Easy to contract dengue & $\begin{array}{l}78(30.1,24.6- \\
36.1)\end{array}$ & $14(23.3,13.4-36.0)$ & 19(19.0, 11.8-28.1) & $45(45.5,35.4-55.8)$ & 0.0001 \\
\hline Not easy to get dengue & $\begin{array}{l}160(61.8,55.6- \\
67.7)\end{array}$ & $46(76.7,64.0-86.6)$ & $66(66.0,55.8-75.2)$ & $48(48.5,38.3-58.8)$ & 0.0010 \\
\hline A severe illness & $\begin{array}{l}130(50.2,43.9- \\
56.4)\end{array}$ & $16(26.2,15.8-39.1)$ & $62(62.0,51.8-71.5)$ & $52(53.1,42.7-63.2)$ & 0.0001 \\
\hline A deadly disease & $\begin{array}{l}102(39.4,33.4- \\
45.6)\end{array}$ & $7(11.7,4.8-22.6)$ & $60(60.0,49.7-69.7)$ & $35(35.4,26.0-45.6)$ & $<0.0001$ \\
\hline Do not know & $21(8.1,5.1-12.1)$ & $0(0,0-6.0)$ & $15(15.0,8.7-23.5)$ & $6(6.1,2.3-12.7)$ & 0.0022 \\
\hline Transmissibility & $\begin{array}{l}198(75.9,70.2- \\
80.9)\end{array}$ & $\begin{array}{l}\text { 60(100.0, 94.0- } \\
100.0)\end{array}$ & $62(62.0,51.8-71.5)$ & $76(76.0,66.4-84.0)$ & $<0.0001$ \\
\hline \multirow{2}{*}{$\begin{array}{l}\text { Directly transmittable from person to } \\
\text { person }\end{array}$} & $N=256$ & $N=61$ & $n=99$ & $N=96$ & \\
\hline & $\begin{array}{l}36(14.1,10.1- \\
18.9)\end{array}$ & $2(3.3,0.4-11.4)$ & $5(5.1,1.7-11.4)$ & $29(30.2,21.3-40.4)$ & $<0.0001$ \\
\hline Heard about dengue & $\begin{array}{l}231(88.5,84.0- \\
92.1)\end{array}$ & $\begin{array}{l}60(100.0,94.0- \\
100.0)\end{array}$ & $84(84.0,75.3-90.6)$ & $87(87.0,78.8-92.9)$ & 0.0060 \\
\hline Knowledge of dengue symptoms & $n=253$ & $n=61$ & $n=100$ & $n=92$ & \\
\hline Fever & $\begin{array}{l}206(81.4,76.0- \\
85.9)\end{array}$ & $\begin{array}{l}61(100.0,94.1- \\
100.0)\end{array}$ & $73(73.0,63.2-81.4)$ & $72(78.2,68.4-82.2)$ & 0.0001 \\
\hline Headache & $\begin{array}{l}177(70.0,63.9- \\
75.5)\end{array}$ & $60(98.4,91.2-100.0)$ & $38(38.0,28.5-48.3)$ & $79(85.9,77.0-92.3)$ & $<0.0001$ \\
\hline Orbital pain & $\begin{array}{l}\text { 65(25.7, 20.4- } \\
31.5)\end{array}$ & $18(29.5,18.5-42.6)$ & $14(14.0,7.9-22.4)$ & $33(35.9,26.1-46.5)$ & 0.0016 \\
\hline Pantalgia & $\begin{array}{l}140(55.3,48.9- \\
61.6)\end{array}$ & $31(50.8,37.7-63.9)$ & $47(47.0,36.9-57.2)$ & $62(67.4,56.8-76.8)$ & 0.0093 \\
\hline Rash & $\begin{array}{l}49(19.4,14.7- \\
24.8)\end{array}$ & $16(26.2,15.8-39.1)$ & $11(11.0,5.6-18.8)$ & $22(23.9,15.6-33.9)$ & 0.0219 \\
\hline Others & $29(11.5,7.8-16.0)$ & $0(0,0-5.9)$ & $28(28.0,19.5-37.9)$ & $1(1.1,0.02-5.9)$ & $<0.0001$ \\
\hline Not know or no response & $8(3.2,1.4-6.1)$ & $0(0,0-5.9)$ & $0(0,0-3.6)$ & $8(8.7,3.8-16.4)$ & 0.0007 \\
\hline Knowledge of dengue causes & $n=259$ & $n=61$ & $n=100$ & $n=98$ & \\
\hline Bacteria & $0(0,0-1.8)$ & $0(0,0-5.9)$ & $0(0,0-3.6)$ & $0(0,0-4.7)$ & - \\
\hline Viruses & $9(3.5,1.6-6.5)$ & $7(11.5,4.7-22.2)$ & $1(1.0,0.03-5.4)$ & $1(1.0,0.03-5.6)$ & 0.0005 \\
\hline Mosquitoes & $\begin{array}{l}213(82.2,77.0- \\
86.7)\end{array}$ & $60(98.4,91.2-100.0)$ & $70(70.0,60.0-78.8)$ & $83(84.7,74.2-89.8)$ & $<0.0001$ \\
\hline Eat improper or dirty food & $5(1.9,0.6-4.4)$ & $0(0,0-5.9)$ & $0(0,0-3.6)$ & $5(5.1,1.7-11.5)$ & 0.0152 \\
\hline Animals & $2(0.8,0.1-2.8)$ & $1(1.6,0.04-8.8)$ & $0(0,0-3.6)$ & $1(1.0,0.03-5.6)$ & - \\
\hline Flies & $0(0,0-1.8)$ & $0(0,0-5.9)$ & $0(0,0-3.6)$ & $0(0,0-4.7)$ & - \\
\hline Be rained or shower with cold water & $0(0,0-1.8)$ & $0(0,0-5.9)$ & $2(0,0-3.6)$ & $0(0,0-4.7)$ & - \\
\hline Others & $0(0,0-5.9)$ & $0(0,0-5.9)$ & $1(1.0,0.03-5.4)$ & $1(1.0,0.03-5.6)$ & - \\
\hline Not know or no response & $36(13.9,9.9-18.7)$ & $0(0,0-5.9)$ & $25(25.0,16.9-34.7)$ & $11(11.2,5.7-19.2)$ & $<0.0001$ \\
\hline
\end{tabular}


Table 2 Demographic characteristics, health perceptions, dengue knowledge and control willingness ( $N=261)$ (Continued)

\begin{tabular}{|c|c|c|c|c|c|}
\hline Variables & $\begin{array}{l}\text { Total No. }(\%, \\
\left.95 \mathrm{Cl}^{\mathrm{a}}\right), n=261\end{array}$ & $\begin{array}{l}\text { No. }(\%, 95 \mathrm{Cl}) \text { in } \\
\text { Manduo, } n=61\end{array}$ & $\begin{array}{l}\text { No. }\left(\%, 95 \mathrm{Cl}^{\mathrm{a}}\right) \text { in } \\
\text { Mandahuo, } n=100\end{array}$ & $\begin{array}{l}\text { No. }\left(\left[\%, 95 \mathrm{Cl}^{\mathrm{a}}\right) \text { in }\right. \\
\text { Jingmenghun, } n=100\end{array}$ & $P$-value \\
\hline \multicolumn{6}{|l|}{ Knowledge of dengue mosquitoes } \\
\hline \multirow[t]{2}{*}{ Piebald or Aedes spp } & $n=255$ & $n=60$ & $n=97$ & $n=98$ & \\
\hline & $\begin{array}{l}194(76.1,70.4- \\
81.2)\end{array}$ & $59(98.3,91.1-100.0)$ & $41(42.3,32.3-52.7)$ & $94(95.9,89.9-98.9)$ & $<0.0001$ \\
\hline Biting time & $n=261$ & $n=61$ & $n=100$ & $n=100$ & \\
\hline $24 \mathrm{~h}$ & $\begin{array}{l}114(43.7,37.6- \\
49.9)\end{array}$ & $56(91.8,81.9-97.3)$ & $25(25.0,16.9-34.7)$ & $34(34.0,24.8-44.2)$ & $<0.0001$ \\
\hline Daytime & $\begin{array}{l}117(44.8,38.7- \\
51.1)\end{array}$ & $5(8.2,2.7-18.1)$ & $54(54.0,43.7-64.0)$ & $58(58.0,47.7-67.8)$ & $<0.0001$ \\
\hline Night & $17(6.5,3.8-10.2)$ & $0(0,0-6.0)$ & $17(17.0,10.2-25.8)$ & $0(0,0-3.6)$ & $<0.0001$ \\
\hline Not know or no response & $14(5.4,3.0-8.8)$ & $0(0,0-6.0)$ & $6(6.0,2.2-12.6)$ & $8(8.0,3.5-15.2)$ & 0.0860 \\
\hline \multicolumn{6}{|l|}{ Aedes larvae habitats } \\
\hline \multirow[t]{2}{*}{ All water sites } & $n=260$ & $n=61$ & $n=99$ & $n=100$ & \\
\hline & $\begin{array}{l}226(86.9,82.2- \\
90.8)\end{array}$ & $60(98.4,91.2-100.0)$ & $68(68.7,58.6-77.6)$ & $98(98.0,93.0-99.8)$ & $<0.0001$ \\
\hline \multirow[t]{2}{*}{ Watered containers or small-scale ponds } & $n=247$ & $n=50$ & $n=97$ & $n=100$ & \\
\hline & $\begin{array}{l}220(89.1,84.5- \\
92.7)\end{array}$ & $\begin{array}{l}50(100.0,100.0- \\
100.0)\end{array}$ & $70(72.2,62.1-80.8)$ & $100(100.0,100.0-100.0)$ & $<0.0001$ \\
\hline $\begin{array}{l}\text { Knowledge regarding reducing Aedes } \\
\text { breeding sites }\end{array}$ & $n=261$ & $n=61$ & $n=100$ & $n=100$ & \\
\hline Maintain sound hygiene & $\begin{array}{l}122(46.7,40.6- \\
53.0)\end{array}$ & $29(47.5,34.6-60.7)$ & $13(13.0,7.1-21.2)$ & $80(80.0,70.8-87.3)$ & $<0.0001$ \\
\hline Turn containers upside down & $\begin{array}{l}242(92.7,88.9- \\
95.6)\end{array}$ & $\begin{array}{l}61(100.0,100.0- \\
100.0)\end{array}$ & $81(81.0,71.9-88.2)$ & $100(100.0,100.0-100.0)$ & $<0.0001$ \\
\hline Drain watered small-scale ponds & $\begin{array}{l}69(26.4,21.2- \\
32.2)\end{array}$ & $9(14.8,7.0-26.2)$ & $5(5.0,3.6-11.3)$ & $55(55.0,44.7-65.0)$ & $<0.0001$ \\
\hline Knowledge of preventing Aedes bites & $n=261$ & $n=61$ & $n=100$ & $n=100$ & \\
\hline Door and window screens & $\begin{array}{l}120(45.9,39.8- \\
52.2)\end{array}$ & $21(34.4,22.7-47.7)$ & $10(10.0,4.9-17.6)$ & $89(89.0,81.2-94.4)$ & $<0.0001$ \\
\hline Use of mosquito coils & $\begin{array}{l}\text { 240(92.0, 88.0- } \\
95.0)\end{array}$ & $60(98.4,91.2-100.0)$ & $80(80.0,70.8-87.3)$ & $100(100.0,100.0-100.0)$ & $<0.0001$ \\
\hline Fogging and spraying with insecticides & $\begin{array}{l}137(52.5,46.2- \\
58.7)\end{array}$ & $28(45.9,33.1-59.2)$ & $18(18.0,11.0-27.0)$ & $91(91.0,83.6-95.8)$ & $<0.0001$ \\
\hline Use of bed nets & $\begin{array}{l}128(49.0,42.8- \\
55.3)\end{array}$ & $9(14.8,7.0-26.2)$ & $29(29.0,20.4-38.9)$ & $90(90.0,82.4-95.1)$ & $<0.0001$ \\
\hline Others & $11(4.2,2.1-7.4)$ & $1(1.6,0.04-8.8)$ & $9(9.0,4.2-16.4)$ & $1(1.0,0-5.5)$ & 0.0099 \\
\hline Not know or no response & $10(3.8,1.9-6.7)$ & $0(0,0-5.9)$ & $10(10.0,4.9-17.6)$ & $0(0,0-3.6)$ & $<0.0001$ \\
\hline Family income source & $n=260$ & & & & \\
\hline Agriculture & $\begin{array}{l}123(41.1,42.8- \\
53.6)\end{array}$ & $1(1.6,0.04-8.8)$ & $31(31.3,22.4-41.4)$ & $91(91.0,83.6-95.8)$ & $<0.0001$ \\
\hline Others & $\begin{array}{l}137(52.7,46.4- \\
58.89)\end{array}$ & $60(98.4,91.2-100)$ & $68(68.7,58.6-77.6)$ & $9(9.0,4.2-16.4)$ & $<0.0001$ \\
\hline Family decision & $n=261$ & & & & \\
\hline Wife or co-decision & $\begin{array}{l}156(59.8,53.5- \\
65.8)\end{array}$ & $39(63.9,50.6-75.8)$ & $48(48.0,37.9-58.2)$ & $69(69.0,59.0-77.9)$ & 0.0167 \\
\hline Husband & $\begin{array}{l}105(40.2,34.2- \\
46.5)\end{array}$ & $22(36.1,24.2-49.4)$ & $52(52.0,41.8-62.1)$ & $31(31.0,22.3-41.0)$ & 0.0167 \\
\hline
\end{tabular}


Table 2 Demographic characteristics, health perceptions, dengue knowledge and control willingness ( $N=261)$ (Continued)

\begin{tabular}{|c|c|c|c|c|c|}
\hline Variables & $\begin{array}{l}\text { Total No. }(\%, \\
\left.95 \mathrm{Cl}^{\mathrm{a}}\right), n=261\end{array}$ & $\begin{array}{l}\text { No. }(\%, 95 \mathrm{Cl}) \text { in } \\
\text { Manduo, } n=61\end{array}$ & $\begin{array}{l}\text { No. }\left(\%, 95 \mathrm{Cl}^{\mathrm{a}}\right) \text { in } \\
\text { Mandahuo, } n=100\end{array}$ & $\begin{array}{l}\text { No. }\left(\left[\%, 95 \mathrm{Cl}^{\mathrm{a}}\right) \text { in }\right. \\
\text { Jingmenghun, } n=100\end{array}$ & $P$-value \\
\hline \multicolumn{6}{|l|}{ Control Willing } \\
\hline \multirow{2}{*}{$\begin{array}{l}\text { Willing to eliminate bamboo and tree } \\
\text { stub holes around houses }\end{array}$} & $n=226$ & $n=32$ & $n=96$ & $n=98$ & \\
\hline & $\begin{array}{l}221(97.8,94.9- \\
99.3)\end{array}$ & $29(90.7,75.0-98.0)$ & $\begin{array}{l}96(100.0,100.0- \\
100.0)\end{array}$ & 96(98.0, 92.8-99.8) & - \\
\hline \multirow{2}{*}{$\begin{array}{l}\text { Willing to clean dumps and turn } \\
\text { containers upside down }\end{array}$} & $n=239$ & $n=40$ & $n=99$ & $n=100$ & \\
\hline & $\begin{array}{l}233(97.5,94.6- \\
99.1)\end{array}$ & 36(90.0, 76.3-97.2) & $\begin{array}{l}99(100.0,100.0- \\
100.0)\end{array}$ & 98(98.0, 93.0-99.8) & 0.0027 \\
\hline $\begin{array}{l}\text { Willing to seek treatment first for } \\
\text { dengue fever from public health } \\
\text { facilities }\end{array}$ & $\begin{array}{l}96(36.8,30.9- \\
42.9)\end{array}$ & $16(26.2,15.8-39.1)$ & $46(46.0,36.0-56.3)$ & $34(34.0,24.8-44.2)$ & 0.5436 \\
\hline
\end{tabular}

${ }^{\mathrm{a}} 95 \% \mathrm{Cl}$ 95\% confidence interval; for all variables, total 261 respondents (61 from Mandou, 100 Mandahuo and 100 Jingmenghun), unless otherwise indicated. Not all subtotals add up to the total of 261 owing to missing values

and another one said "The water is very important for human health because people have to drink water every day. The dirty water may cause diseases". In Jingmenghun, one of the two rural communities, one key informant noted "Fresh air is good for health. The fewer trees lead to the poorer air, and then the poorer air may cause diseases". As shown in Table 2, only about onethird of the participants $(29.7 \%, 77 / 259)$ agreed to that poverty was one of disease causes. A significantly higher proportion of the participants $(54.0 \%, 54 / 100)$ in Jingmenghun, one of the two rural communities, agreed to that poverty was one of disease causes compared to those in the urbanized community $(11.7 \%, 7 / 60)$ and Mandahuo, another one $(16.2 \%, 16 / 99)$ of the two rural communities. In Jingmenghun, one of the key informants noted "The poor may be unhappy, malnutrition and unable to go to see doctors in time".

\section{Dengue fever knowledge}

As shown in Table 2, most of the participants $(88.5 \%$, 231/256) heard about dengue. Many of them $(81.4 \%$, 2061/253) were aware that fever was a primary symptom of dengue fever. More than half of the participants $(55.3 \%, 140 / 253)$ reported pantalgia. Less than one-third of the participants were aware that orbital pain $(25.7 \%$, $65 / 253)$ in the eyes and rashes $(19.4,49 / 253)$ were ones of dengue fever symptoms. Just one of the key informants did not reported that fever was one of dengue fever symptoms. More than one-third key informants $(38.9 \%, 7 / 18)$ did not know more specific symptoms of dengue fever such as pantalgia, rashes and orbital pain in the eyes. Only five of the key informants mentioned that rashes were one of dengue fever symptoms, and two of the key informants mentioned orbital pain in the eyes. About half of the quantitative study participants (50.2\%, $130 / 259)$ were aware that dengue fever was a severe illness. More than one-third of the participants (39.4\%, $102 / 259$ ) were aware that dengue fever was a deadly disease. A significantly lower percentage of the participants in the urbanized community $(26.2 \%, 16 / 60)$ had the perceived severity of dengue fever compared to those in the border community (Mandahuo, 62.0\%, 62/100). Most of the key informants $(88.9 \%, 16 / 18)$ perceived that dengue fever was a severe or deadly disease. One of the key informants in the urbanized community noted "No deaths occur in Jinghong City, but heard about deaths in Laos and Myanmar. If a dengue fever patient did not receive treatment in time, he or she might become severe or even death". One of key informants in the border community said "If someone contracted dengue fever for the second time, the disease could be severer than the first attack". One of key informants in the community with only one imported dengue fever case (Jingmenghun) said "Dengue fever is a severe disease, especially in the first seven days of the illness. Dengue fever is a transmittable disease and it may cause social panic when it becomes epidemic". However, one of key informants in border community (Mandahu) noted "Dengue fever should not be a dangerous disease in modern medical situation. Dengue fever is curable". One of key informants in the community with only one imported dengue fever case said "Dengue fever cannot lead to a death. One of my neighbors contracted dengue fever and was cured in the Menghai County Hospital".

More than three-quarters of the participants $(75.9 \%$, 198/259) knew that dengue fever was an infectious disease. A small part of the participants $(14.1 \%, 36 / 256)$ thought that dengue fever was able to directly transmit from person to person by speaking, breathing and physical contact. As shown in Table 2, a significantly higher percentage of the participants in the urbanized community $(100 \%, 60 / 60)$ knew that dengue fever was transmittable compared to those in the two rural communities (Mandahuo 62\%, 62/100 and Jingmenghun 53.1\%, 52/ 99). Many of the participants $(82.2 \%, 213 / 259)$ knew that mosquitoes transmitted dengue fever. However, only a 
few of participants $(3.5 \%, 9 / 259)$ knew that a virus caused dengue fever. A significantly higher proportion of participants in the urbanized community $(98.4 \%, 60 / 61)$ knew that mosquitoes transmitted dengue fever compared to those in the two rural communities (Mandahuo $70 \%, 70 / 100$ and Jingmenghun 84.7\%, 83/98). Most of the key informants $(77.8 \%, 14 / 18)$ confirmed that dengue virus was only transmitted by mosquitoes. However, one of the key informants in the community with only one imported dengue fever case) noted "Toxins cause dengue fever. People would be ill if there are toxins in their bodies. The toxins can be transmitted by physical contacts and breathing". All six key informants in the border community agreed that in Dai ethnic language dengue fever was called "Paya Yong", in which "Paya" was illness and "Yong" was mosquitoes. This Dai ethnic name for dengue fever suggested that dengue fever was associate with mosquitoes in Dai ethnic language. However, all 12 key informants in the two non-border communities did not agree that there was a Dai ethnic language name for dengue fever. This may imply that dengue fever in culture is also an imported disease among Dai people in China.

Most of the participants $(76.1 \%, 194 / 255)$ knew that dengue vector was Aedes sp or piebald mosquitoes. Less than half of the participants $(44.8 \%, 117 / 261)$ were aware that Aedes $s p$ bit people in daytime. Many of the participants $(92.0 \%, 240 / 261)$ reported that use of mosquito coils could effectively prevent bite of Aedes $s p$. Most of the participants $(89.1 \%, 220 / 260)$ were aware that Aedes $s p$ bred in containers or small-scale pond with clean and stagnant water. A lot of the participants (92.7\%, 242/ 261) agreed that turning containers upside down could reduce number of Aedes mosquitoes to prevent dengue fever (Table 2). Similar results were obtained during indepth interview. One of the key informants noted "Ponded water between houses is easy to breed Aedes sp in raining season. However, it is difficult to clear the ponded water". Most of the key informants (94.4\%, 17/ 18) agreed that breeding site management could reduce number of Aedes spp mosquitoes to prevent dengue fever. All six key informants in the urbanized community emphasized environmental management as importance to control of Aedes spp mosquitoes compared to those in the two rural communities.

\section{Control willingness and related factors}

As Table 2 shows, most of the participants (97.8\%, 221/ 226) indicated their willingness to eliminate bamboo and tree stub holes around their houses. When all participants were asked if they would consider turning containers upside down, $97.5 \%$ (233/239) of them answered "yes". However, this answer was not fully consistent with results of the qualitative study. Results of the in-depth interview conducted in the urbanized community indicated that the local government has enforced environmental management to control Aedes mosquitoes. A village leader introduced "The terms of 'The Villager Convention of Manduo' request all families to clean their houses and surrounding environment every day. The team of village leaders conducts two rounds of supervision-visits every week. If Aedes sp larvae were found in a household, the family should pay a fine of CNY500. If Aedes sp larvae were found again in the same household, the fine would increase to CNY1000. If any household did not comply with the terms of the Villager Convention, we could stop supply of the pipe water and the electricity". Results of the in-depth interview conducted in the two rural communities indicated that their fellow villagers did not like to clean and maintain environmental hygiene. One of the key informants in the community with only one imported dengue fever case noted "Some villagers have not recognized the severity of dengue fever. They do not like to clean their houses and surrounding environment. We need health education to promote their knowledge, awareness and action". Results of multivariate logistic analysis (MLA) showed that the participants with FWI 4-5 [odd ratio (OR) 22.9728, 95\% confidence interval (CI): 2.4257-217.5688, $p=0.0063$ ] were more likely to turn containers upside down compared to those with FWI 1-3; and the participants in the urban community (OR: 0.0239, CI: 0.00190.3032, $p=0$. 0.0040) were less inclined to turn containers upside down compared to those in the two rural communities (Table 3).

When asked where they or their family members would consider to seek treatment if they had an attack of dengue fever, nearly two-third of participants $(63.2 \%$, $165 / 239$ ) reported that they would buy drugs from drugstores first, only about one-third of them (36.8\%, 96/ 239) answered they would go to a public health facility first (Table 2). Results of the qualitative data analysis indicated that Dai people liked to take over-the-counter (OCT) drugs for disease treatment first. The qualitative data analysis summarized out three reasons for this treatment-seeking intention: 1) High accessibility of OCT drugs, one of the key informants said "There are a lot of drugstores anywhere, from the city to the countryside. We can buy most of drugs that we need at any time. The prices of store drugs are much cheaper than hospital drugs". 2) A long time needs to seek treatment from public hospitals, one of the key informants noted "Travel needs time; waiting to see a doctor needs time". 3) They have to pay for some unnecessary laboratory tests or physical examinations, one of the key informants noted "The hospital will request us to do a set of laboratory tests or physical examinations. Some of laboratory tests or physical examinations are not actually essential, 
Table 3 Factors associated with environmental management willingness for vector control $(N=261)$

\begin{tabular}{|c|c|c|c|c|c|}
\hline Independent variables & No. respondents of WTCUD $(\%, 95 \mathrm{Cl})$ & Crude $\mathrm{OR}^{\mathrm{b}}\left(95 \% \mathrm{Cl}^{\mathrm{c}}\right)$ & $P$ values & Adjusted $\mathrm{OR}^{\mathrm{b}}\left(95 \% \mathrm{Cl}^{\mathrm{c}}\right)$ & $P$ values \\
\hline \multicolumn{6}{|l|}{ Location of villages } \\
\hline Jinghong $(n=40)$ & $36(90.0,76.3-97.2)$ & $0.0914(0.0161-0.5176)$ & 0.0068 & $0.0239(0.0019-0.3032)$ & 0.0040 \\
\hline Menghai ( $n=199$ ) & $197(99.0,96.4-99.9)$ & 1 & & 1 & \\
\hline \multicolumn{6}{|l|}{ Sex of participants } \\
\hline Female $(n=165)$ & $146(96.7,92.4-98.9)$ & $0.3356(0.0386-2.9202)$ & 0.3226 & & \\
\hline Male $(n=96)$ & $87(98.9,93.8-100)$ & 1 & & & \\
\hline \multicolumn{6}{|c|}{ Age of participants (years) } \\
\hline $17-40(n=124)$ & $39(97.5,86.8-99.9)$ & $0(0->1.0 \mathrm{E} 12)$ & 0.9626 & & \\
\hline $41-84(n=137)$ & $127(100,97.1-100)$ & 1 & & & \\
\hline \multicolumn{6}{|l|}{ School education } \\
\hline$>6(n=132)$ & $36(100,90.3-100)$ & $0.1852(0.0213-1.6091)$ & 0.1263 & $0.4364(0.0371-5.1366)$ & 0.9963 \\
\hline$\leq 6(n=129)$ & $121(99.2,95.5-100)$ & 1 & & 1 & \\
\hline \multicolumn{6}{|l|}{ Family wealth index } \\
\hline $4-5(n=246)$ & $115(99.1,95.3-100)$ & $10.0371(1.6559-60.8373)$ & 0.0121 & $22.9728(2.4257-217.5688)$ & 0.0063 \\
\hline $1-3(n=13)$ & $11(84.6,54.6-98.1)$ & 1 & & 1 & \\
\hline \multicolumn{6}{|c|}{ Belief of Buddha blessing good people } \\
\hline Yes $(n=181)$ & $178(98.3,95.2-99.7)$ & $3.2364(0.6353-16.4873)$ & 0.1574 & $2.8571(0.4992-16.3514)$ & 0.2382 \\
\hline No $(n=58)$ & $55(94.8,85.6-95.9)$ & 1 & & 1 & \\
\hline \multicolumn{6}{|c|}{ Belief of all natural factors influencing human health } \\
\hline Yes $(n=177)$ & $171(96.6,92.8-98.8)$ & Undefined OR & 0.9620 & & \\
\hline No $(n=61)$ & $61(100.0,100.0-100.0)$ & 1 & & & \\
\hline \multicolumn{6}{|c|}{ Belief of sound hygiene helpful to people's health } \\
\hline Yes $(n=199)$ & $194(97.5,94.2-99.2)$ & $0.9949(0.1131-8.7512)$ & 0.9963 & & \\
\hline No $(n=40)$ & $39(97.5,86.8-99.9)$ & 1 & & & \\
\hline \multicolumn{6}{|c|}{ Respondents have heard about dengue } \\
\hline Yes $(n=210)$ & $204(97.1,93.9-98.9)$ & Undefined OR & 0.9739 & & \\
\hline No $(n=29)$ & $29(100.0,100.0-100.0)$ & 1 & & & \\
\hline \multicolumn{6}{|c|}{ Perceive dengue as a severe or deadly disease } \\
\hline Yes $(n=101)$ & $96(95.1,88.8-95.4)$ & $0.1422(0.0164-1.2360)$ & 0.0771 & $0.1830(0.0202-1.6549)$ & 0.1306 \\
\hline No $(n=136)$ & 135(99.3, 96.0-100.0) & 1 & & 1 & \\
\hline \multicolumn{6}{|c|}{ Perceived easy to be infected by dengue } \\
\hline Yes $(n=74)$ & $71(96.0,88.6-99.2)$ & $0.4438(0.0874-2.2518)$ & 0.3269 & & \\
\hline No $(n=163)$ & $160(98.2,94.7-99.6)$ & 1 & & & \\
\hline \multicolumn{6}{|c|}{ Know dengue only transmitted by mosquitoes } \\
\hline Yes $(n=61)$ & $60(98.4,91.3-100.0)$ & $1.7733(0.2034-15.4582)$ & 0.6041 & & \\
\hline No $(n=177)$ & $172(97.2,93.5-99.1)$ & 1 & & & \\
\hline \multicolumn{6}{|c|}{ Know dengue mosquitoes biting in daytime or whole day } \\
\hline Yes $(n=208)$ & 203(97.6, 94.5-99.2) & $1.4000(0.1579-12.4093)$ & 0.7625 & & \\
\hline No $(n=30)$ & 29(96.7, 82.8-99.9) & 1 & & & \\
\hline \multicolumn{6}{|c|}{ Know at least one breeding site of Aedes spp larvae } \\
\hline Yes $(n=208)$ & 203(97.6, 94.5-99.2) & Undefined OR & 0.9678 & & \\
\hline No $(n=27)$ & $27(100.0,100.0-100.0)$ & 1 & & & \\
\hline \multicolumn{6}{|c|}{ Know at least one method of mosquito adult control } \\
\hline Yes $(n=83)$ & $82(98.8,93.5-100.0)$ & Undefined OR & 0.9711 & & \\
\hline
\end{tabular}


Table 3 Factors associated with environmental management willingness for vector control $(N=261)$ (Continued)

\begin{tabular}{|c|c|c|c|c|c|}
\hline Independent variables & No. respondents of WTCUD ${ }^{a}(\%, 95 \mathrm{Cl})$ & Crude $\mathrm{OR}^{\mathrm{b}}\left(95 \% \mathrm{Cl}^{\mathrm{c}}\right)$ & $P$ values & Adjusted $\mathrm{OR}^{\mathrm{b}}\left(95 \% \mathrm{Cl}^{\mathrm{c}}\right)$ & $P$ values \\
\hline No $(n=10)$ & $10(100.0,100.0-100.0)$ & 1 & & & \\
\hline \multicolumn{6}{|c|}{ Know at least one method of mosquito larva control } \\
\hline Yes $(n=128)$ & $122(95.3,90.2-98.3)$ & Undefined OR & 0.9695 & & \\
\hline No $(n=7)$ & $7(100.0,100.0-100.0)$ & 1 & & & \\
\hline
\end{tabular}

${ }^{a}$ WTCUD Willing to turn containers upside down, ${ }^{b}$ OR Odds ratio, ${ }^{c} 95 \% \mathrm{Cl} 95 \%$ confidence interval. For all variables, total 261 respondents, unless otherwise indicated. Not all subtotals add up to the total of 261 owing to missing values

but we still have to pay for the unnecessary laboratory tests or physical examinations, in spite of Xin Nong He (new rural health insurance in China) can cover parts of the money". In the MLA, the participants with the perceived severity of dengue fever (OR: 5.0564 , CI: $2.0672-$ 12.3683, $p=0$. 0.0004) compared to those without the perceived severity and the participants with the awareness of sound hygiene (OR: 11.5671, CI: 2.0672-12.3683, $p=0.0 .0055)$ compared to those without awareness of sound hygiene tended to seek treatment first from public health facilities. Suspected dengue fever patients from households with wife or co-decision (OR: 11.5671, CI: 2.0672-12.3683, $p=0$. 0.0055) were less likely to seek treatment first from public health facilities compared to those in households with husband decision (Table 4).

\section{Discussion \\ Health perceptions, dengue knowledge and control willingness}

The Shan people in Myanmar and the Dai people in China are the same ethnicity. Just like the Shan people in Myanmar [8], vast majority of Dai people are Buddhists. Most of the participants in the two rural communities (Mandahu and Jingmenghun) believed that good deeds come back to help themselves, and evil deeds haunt themselves. In spite of vast majority of the participants in the urbanized community (Manduo) were Buddhists, they did not believed that the Lord Buddha would protect good people any more. Instead, a significantly higher proportion of the participants in the urbanized community perceived that people's diseases were associated with the poor hygiene and natural factors compared to those participants in the two rural communities. Taken overall, knowledge of dengue fever was sound among the Dai people. As shown in the investigations of the Shan people [20] and the Kachin people [21] in Myanmar, knowledge level of dengue fever are associated with dengue fever incidence. In this study, the knowledge level of dengue fever in the two communities with dengue fever outbreaks (Manduo and Mandahuo) was significantly higher than the community with only one imported dengue fever case (Jingmenghun) in 2017. The perceived susceptibility and the perceived severity of dengue fever were low among the Dai people. Only a half of the participants perceived dengue fever as a severe disease, and less than one-third of them agreed that they were easy to contract dengue fever. A significantly lower percentage of the participants in the urbanized community had the perceived susceptibility and the perceived severity of dengue fever compared to those in the two rural communities. The participants reported a high willingness of vector control, with vast majority of the participants expressing willingness to eliminate bamboo and tree stub holes, and turning containers upside down. However, a significantly lower percentage of the participants considered to seek treatment first for dengue fever from public facilities. In current study, a significantly lower proportion of the participants in the urbanized community expressed the willingness of vector control and treatment-seeking willingness from public facilities for dengue fever compared to those in the two rural communities. The basic concepts of the HBM are perceived susceptibility, perceived severity, perceived benefits, perceived barriers to taking health action, cues to action and self-efficacy $[16,17]$. Wong et al. reported that some heath beliefs could help to prevent dengue fever [20]. An investigation in Cambodia argued that knowledge was unlikely to have any significant effect on practices in its setting [11]. These concepts interpreted why the participants in the urbanized community had a higher knowledge level of dengue fever, but a significantly lower proportion of dengue control willingness compared to those in the two rural communities. Traditional beliefs of the Dai people have changed with the urbanization, and the improved medication has reduced their perceived susceptibility and severity to dengue fever in China. Dengue fever control at the outskirts of cities is a great challenge in the world [10, 22]. Just like the urbanized community of this study, outbreaks of dengue fever were more frequently occurred in cities in China [4-6]. The results of this study indicated the association between dengue control willingness, health belief, perceived susceptibility and perceived severity. The enforced environmental management has been used to control Aedes spp in the three study sites, however, the intervention efficacy seems limited. Thus, the Dai people still need more effective 
Table 4 Factors associated with seeking treatment first for dengue fever from public health facilities $(N=261)$

\begin{tabular}{|c|c|c|c|c|c|}
\hline Independent variables & $\begin{array}{l}\text { No. respondents of } \\
\text { WSTPHF }^{\mathrm{a}}(\%, 95 \mathrm{Cl})\end{array}$ & Crude $\mathrm{OR}^{\mathrm{b}}\left(95 \% \mathrm{Cl}^{\mathrm{c}}\right)$ & $P$ values & $\begin{array}{l}\text { Adjusted } \mathrm{OR}^{\mathrm{b}} \\
\left(95 \% \mathrm{Cl}^{\mathrm{C}}\right)\end{array}$ & $P$ values \\
\hline \multicolumn{6}{|l|}{ Location of villages } \\
\hline Jinghong $(n=61)$ & $16(26.2,15.8-39.1)$ & $0.5335(0.2822-1.0085)$ & 0.0531 & $0.8816(0.1977-3.931)$ & 0.8688 \\
\hline Menghai $(n=200)$ & $807(40.0,33.2-47.2)$ & 1 & & 1 & \\
\hline \multicolumn{6}{|l|}{ Sex of participants } \\
\hline Female $(n=165)$ & $67(40.6,33.0-48.5)$ & $1.5795(0.9250-2.6971)$ & 0.0941 & $1.6107(0.7911-3.279)$ & 0.1889 \\
\hline Male $(n=96)$ & $29(30.2,21.3-40.4)$ & 1 & & 1 & \\
\hline \multicolumn{6}{|l|}{ Age of participants (years) } \\
\hline $17-40(n=124)$ & $48(38.7,30.1-47.9)$ & $1.1711(0.7076-1.9380)$ & 0.5390 & & \\
\hline $41-84(n=137)$ & $48(35.0,27.1-43.7)$ & 1 & & & \\
\hline \multicolumn{6}{|l|}{ School education } \\
\hline$>6(n=132)$ & $54(40.9,32.4-49.8)$ & $1.3378(0.7470-2.3959)$ & 0.3277 & & \\
\hline$\leq 6(n=129)$ & $42(32.6,24.6-41.5)$ & 1 & & & \\
\hline \multicolumn{6}{|l|}{ Family wealth index } \\
\hline $4-5(n=246)$ & $90(36.6,30.6-42.9)$ & $0.6731(0.2194-2.0646)$ & 0.4888 & & \\
\hline $1-3(n=13)$ & $100(100,75.3-100)$ & 1 & & & \\
\hline \multicolumn{6}{|c|}{ Belief of Buddha blessing good people } \\
\hline Yes $(n=182)$ & $73(40.1,32.9-47.6)$ & $1.6014(0.9058-2.8313)$ & 0.1053 & $0.9937(0.2965-3.3300)$ & 0.9918 \\
\hline No $(n=78)$ & $23(29.5,19.7-40.9)$ & 1 & & 1 & \\
\hline \multicolumn{6}{|c|}{ Belief of all natural factors influencing human health } \\
\hline Yes $(n=198)$ & $66(33.8,27.3-40.9)$ & $0.5644(0.3152-1.0104)$ & 0.9620 & & \\
\hline No $(n=61)$ & $29(47.5,34.6-60.7)$ & 1 & & & \\
\hline \multicolumn{6}{|c|}{ Belief of sound hygiene helpful to people's health } \\
\hline Yes $(n=210)$ & $89(42.4,35.6-49.4)$ & $3.778(1.5204-9.3888)$ & 0.0042 & $11.5671(2.0505-65.2502)$ & 0.0055 \\
\hline No $(n=50)$ & $7(14.0,5.8-26.7)$ & 1 & & 1 & \\
\hline \multicolumn{6}{|l|}{ Heard about dengue } \\
\hline Yes $(n=231)$ & $90(39.0,32.6-45.6)$ & $\underline{2.5532} \underline{(1.0045-\underline{6.4896)}}$ & $\underline{0.0489}$ & $1.2562(0.2348-6.7201)$ & 0.7898 \\
\hline No $(n=30)$ & $6(20.0,7.7-38.6)$ & 1 & & 1 & \\
\hline \multicolumn{6}{|c|}{ Perceive dengue as a severe disease } \\
\hline Yes $(n=130)$ & $67(51.5,42.6-60.4)$ & $4.0176(2.3270-6.9366)$ & $<0.0001$ & $5.0564(2.0672-12.3683)$ & 0.0004 \\
\hline No $(n=129)$ & $27(20.9,14.3-29.0)$ & 1 & & 1 & \\
\hline \multicolumn{6}{|c|}{ Know fever as one of dengue symptoms } \\
\hline Yes $(n=206)$ & $83(40.3,33.5-47.3)$ & $2.2056(1.0627-4.5775)$ & 0.0337 & $1.4204(0.4018-5.0219)$ & 0.5860 \\
\hline No $(n=46)$ & $11(23.9,12.6-38.8)$ & 1 & & 1 & \\
\hline \multicolumn{6}{|l|}{ Perceive dengue transmissible } \\
\hline Yes $(n=198)$ & $76(38.4,31.6-45.5)$ & $1.3393(0.7329-2.4474)$ & 0.3422 & & \\
\hline No $(n=63)$ & $20(31.8,20.6-44.7)$ & 1 & & & \\
\hline \multicolumn{6}{|l|}{ Family income source } \\
\hline Agriculture $(n=123)$ & $45(36.6,28.1-45.8)$ & $1.0038(0.6055-1.6643)$ & 0.9881 & & \\
\hline Others $(n=137)$ & $50(36.5,28.4-45.2)$ & 1 & & & \\
\hline \multicolumn{6}{|l|}{ Family decision } \\
\hline Wife or co-decision $(n=156)$ & $45(28.9,21.9-36.6)$ & $0.4293(0.2562-0.7193)$ & 0.0013 & $0.3505(0.1682-0.7305)$ & 0.0051 \\
\hline Husband $(n=105)$ & $51(48.6,38.7-58.5)$ & 1 & & 1 & \\
\hline
\end{tabular}

${ }^{\mathrm{a} W S T P H F}$ Willing to seek treatment first from public health facilities, ${ }^{\mathrm{b}} \mathrm{OR}$ Odds ratio, ${ }^{\mathrm{c}} 95 \% \mathrm{Cl} 95 \%$ confidence interval. For all variables, total 261 respondents, unless otherwise indicated. Not all subtotals add up to the total of 261 owing to missing values 
health education interventions about the susceptibility, severity and control willingness of dengue fever.

\section{Barriers of vector control willingness}

The breeding sites of Aedes spp mosquitos are small water ponds, containers and discarded tires with clean and stagnant water. Living environmental management of Aedes spp mosquitoes is thereby becoming the most critical strategy for dengue vector control [10, 23]. Elimination of Aedes spp habitats at residents' houses and yards needs the community participation. The HBM regards that health-related action depends upon the simultaneous occurrence of three categories of factors, i.e. sufficient motivation, perceived threat and costbeneficial [16]. In this study, the results of MLA indicated that the participants with FWI 4-5 were more likely to turn containers upside down compared to those with FWI 1-3. This might be due to the fact that the wealthier participants had a better understanding of knowledge provided about environmental management to prevent dengue fever. Just like the discussion above, changed health beliefs and reduced perception of the severity and the susceptibility of dengue fever might be the reason why the participants in the urban community were less inclined to turn containers upside down compared to participants in the two rural communities. The participants in the urban community had the highest level of dengue knowledge among the three study sites, however, they had the lowest willingness of vector control. This might be the fact that they have become ennui to the frequent request of enforced environmental management. This finding reveals that more effective health education interventions are essential to promote the Dai people's perceived severity, perceived susceptibility of dengue fever, and sufficient motivation of vector control.

\section{Barriers of appropriately treatment-seeking willingness for dengue fever}

Appropriately treatment-seeking willingness is essential for surveillance and response of infectious diseases. Suspected dengue patients have intention to seek proper medical care early can reduce the complication of dengue fever, and further reduce transmission of dengue virus $[24,25]$. Due to the fact that laboratory test for dengue fever is only available in the public health facilities in China, it is expected that suspected dengue patients should seek treatment first from the public health facilities. Results of the quantitative study indicated that just about one-third of the participants expressed their willingness to seek treatment first for dengue fever from the public health facilities. The MLA identified that the participants with the perceived severity and the participants with the awareness of sound hygiene tended to seek treatment first from public health facilities compared to participants without the perceived severity and the awareness of sound hygiene. This might be the fact that the participants with the perceived severity and the awareness of sound hygiene had a better understanding of information provided about availability of laboratory test for dengue fever in the public health facilities, and the importance of prompt diagnosis and early treatment to relieve symptoms of dengue fever and to reduce related complication. This study suggests that availability of laboratory test for dengue fever in the public health facilities should be focus information to the public. It may be, therefore, worthwhile trialing targeted educational intervention to promote the Dai people's awareness of the severity and knowledge about dengue fever. The reason why a significantly lower proportion of the participants from the households with wife or codecision had intention to seek treatment first for dengue fever from the public health facilities compared to those participants from the households with husband decision, is not clear, thus, future investigation should be essential. Findings of the qualitative study revealed that the health authority needs to address a number of important issues on the Dai people's treatment-seeking willingness, including overuse of OCT drugs, accessibility of laboratory diagnosis, time cost and financial expenditure of diagnosis and treatment service for dengue fever in the public health facilities.

\section{Challenges of dengue fever control}

In Yunnan, the first locally infected dengue fever case was confirmed in 2013 [6], shortly after the successful control of malaria along China-Myanmar border [26]. The pattern of "big year" and "small year" of dengue fever incidence appeared in Yunnan since 2013. The "big year" means a lot number of dengue fever outbreaks in a year, and the "small year" means few outbreaks with a few dengue fever cases within a year. The year after a "big year" was often a "small year" and a "big year" would be found subsequently after a "small year" [27]. Why this phenomenon of "big year" and "small year" for dengue fever appeared is still unknown. The intervention intensity and the local people's control willingness might be an explanation for this phenomenon. The asymptomatic dengue virus infection was estimated accounting for more than $75 \%$ of overall dengue virus infection in the world $[28,29]$. In China, when an outbreak happens, the environmental management for controlling Aedes spp mosquitoes was enforced by social mobilization and governmental regulations. However, the intervention intensity is usually reduced when without outbreaks and with reduced number of dengue fever cases. The phylogenetic study revealed the high virus relatedness between dengue outbreaks and imported dengue virus in Yunnan [7]. Year 2017 was a "big year" with many breaks, and 2018 
was a "small year" without any outbreak, and then 2019 was a "big year" with many outbreaks again. No any outbreak was found in 2020, and only a few of dengue fever cases was detected in the first half year of 2021 due to border limitation for COVID-2019 control. This reveals that dengue fever situation in Yunnan each year may be associated with imported dengue virus and intervention intensity. This finding suggests that people's control willingness could help to reshape focus messages and approach of health education interventions.

\section{Limitations}

The primary limitation is that this study is quantitatively dominated, using qualitative data to triangulate the quantitative results and to explore reasons for the quantitative results. The depth off qualitative study is not enough to explore causes of existing health perceptions, dengue knowledge, and control willingness. Among the second limitation of this study, a lower response rate of the participants to the questions of willingness in the urbanized community might lead to information bias.

\section{Conclusion}

In conclusion, the health perceptions of Dai people are changing with ongoing urbanization. Most Dai people have sound knowledge, but a low proportion of the participants with the perceived susceptibility and the severity of dengue fever. The willingness of the Dai people in environmental management for vector control and seeking appropriate treatment for dengue fever are two main challenges in dengue fever control. This suggests the need to provide information of laboratory test availability in the public health facilities, and health educational interventions should target to promote the perceived susceptibility and the perceived severity of dengue fever among the Dai people.

\section{Abbreviations \\ WHO: World Health Organization; HBM: Health belief model; TCUD: Turn containers upside down; WTCUD: Willing to turn containers upside down; WSTPHF: Willing to seek treatment first from public health facilities; OCT: Over-the-counter; 95\% Cl: Confidence interval; OR: Odds ratio; FWI: Family wealth index}

\section{Supplementary Information}

The online version contains supplementary material available at https://doi. org/10.1186/s12889-021-11864-9.

\section{Additional file 1.}

Additional file 2.

\section{Acknowledgements}

We thank all participants for their contribution of time and patience in the study. We would like to thank Ms Zhuang Yu from the Health Post of Mandou Village Committee in Jinghong City, Mr Yingxiang Ai from the Daluo Town Hospital and Ms Jiaonan Yu from the Health Post of Jingmenghun Village Committee in Menghai County for their actively participating the field survey, Ms Li Huiqiong from Yunnan Simao Sanmu Group for the entry of the data. We thank Dr. Hongzhang Xu from the Fenner School of Environment and Society at the Australian National University for his comments and copyediting English language. The opinions expressed are those of the authors and do not necessarily reflect those of the above-mentioned organizations and people.

\section{Authors' contributions}

$\mathrm{HL}$ and J-WX conceived the study and developed the protocol, analyzed and interpreted the data, conducted the field survey, and wrote the first draft of the paper. CJF conducted the field survey and provided critical comments. All authors read and approved the final manuscript.

\section{Funding}

The project was funded by the National Social Science Fund of China (16ASH004). JWX received the grant. Funder's website: http://www.npopss$\mathrm{cn} . \mathrm{gov} . \mathrm{cn} /$. The funders had no role in study design, data collection and analysis, decision to publish, or preparation of the manuscript.

\section{Availability of data and materials}

All relevant data are within the manuscript. The datasets are available from the corresponding author on reasonable request.

\section{Declarations}

\section{Ethics approval and consent to participate}

The ethical approval was granted by the Ethics Committee of the Yunnan Institute of Parasitic Diseases in China (ECYIPD201705). Verbal consent was approved by the Ethics Committee as an acceptable form, as the study was interview-based and did not include any human specimens. The study was also approved by the Health Commission of the Xishuangbannan Prefecture, Health Bureau of the Jinghong City and Menghai County. All study participants were adults. According to the World Medical Association Declaration of Helsinki, the purpose and procedures of the study were disclosed and explained to the participants before obtaining their consent. Participation was entirely voluntary, and participants could pass on a question, take a break or withdraw their consent from the study without providing any explanation at any time. Their continued consent was assumed if they did not refuse to answer questions. The investigators will grant the confidentiality of participants and their responses.

\section{Consent for publication}

The authors give consent for publication.

\section{Competing interests}

The authors declare that they have no conflict of interest.

\section{Author details}

${ }^{1}$ Yunnan Institute of Parasitic Diseases, Yunnan Provincial Key Laboratory of Vector-borne Diseases Control and Research, Xiyuan Road, Pu'er City 665000, China. ${ }^{2}$ Institute of Pathogens and Vectors, Basic Medical College, Dali University, Wanhua Road, Xiaguang District, Dali City 671000, China. ${ }^{3}$ Pu'er Blood Bank, Chayuan Road, Pu'er City 665000, Yunnan Province, China.

Received: 30 September 2020 Accepted: 27 September 2021 Published online: 12 October 2021

\section{References}

1. WHO. Dengue control. Geneva: World Health Organization. http://www who.int/denguecontrol/epidemiology/en/ Accessed 1 March 2019.

2. LNdS O, Itria A, Lima EC. Cost of illness and program of dengue: $\mathrm{A}$ systematic review. PLoS One. 2019;14(2):e0211401.

3. Shepard DS, Undurraga EA, Halasa YA, Stanaway JD. The global economic burden of dengue: a systematic analysis. Lancet Infect Dis. 2016;16(8):93541. https://doi.org/10.1016/S1473-3099(16)00146-8.

4. Lai S, Huang Z, Zhou H, Anders K, Perkins TA, Yin W, et al. The changing epidemiology of dengue in China, 1990-2014: a descriptive analysis of 25 years of nationwide surveillance data. BMC Med. 2015;13(1):100. https://doi. org/10.1186/s12916-015-0336-1.

5. Ooi EE. The re-emergence of dengue in China. BMC Med. 2015;13(1):99. https://doi.org/10.1186/s12916-015-0345-0. 
6. Yang MD, Jiang JY, Guo XF, Wu C, Zhou HN. Epidemiological analysis and investigation of dengue fever in Yunnan Province from 2009 to 2014. J Pathogen Bio. 2015;10(8):738-42 (in Chinese).

7. Wang B, Li Y, Feng Y, Zhou H, Liang Y, Dai J, et al. Phylogenetic analysis of dengue virus reveals the high relatedness between imported and local strains during the 2013 dengue outbreak in Yunnan, China: a retrospective analysis. BMC Infect Dis. 2015;15(1):142. https://doi.org/10.1186/s12879-0150908-X.

8. Xu JW, Liu H, Ai Z, Yu Y, Yu B. The Shan people's health beliefs, knowledge and perceptions of dengue in eastern Shan special region IV, Myanmar. PLoS Negl Trop Dis. 2019;13(6):e0007498. https://doi.org/10.1371/journal. pntd.0007498

9. Villar L, Dayan GH, Arredondo-GarcõÂa JL, et al. Efficacy of a tetravalent dengue vaccine in children in Latin America. N Engl J Med. 2015;372(2): 113-23. https://doi.org/10.1056/NEJMoa1411037.

10. WHO. Dengue Guidelines for diagnosis, treatment, prevention and control Geneva: World Health Organization; 2009. accessed 27 May 2016

11. Kumaran E, Doum D, Keo V, Sokha L, Sam B, Chan V, et al. Dengue knowledge, attitudes and practices and their impact on community-based vector control in rural Cambodia. PLoS Negl Trop Dis. 2018;12(2):e0006268. https://doi.org/10.1371/journal.pntd.0006268.

12. Lin H, Liu T, Song T, Lin L, Xiao J, Lin J, et al. Community involvement in dengue outbreak control: an integrated rigorous intervention strategy. PLoS Negl Trop Dis. 2016;10(8):e0004919. https://doi.org/10.1371/journal.pntd. 0004919.

13. McNaughton D. The importance of long-term social research in enabling participation and developing engagement strategies for new dengue control technologies. PLoS Negl Trop Dis. 2012;6(8):e1785. https://doi.org/1 0.1371/journal.pntd.0001785.

14. Liu H, Xu JW, Ai Z, Yu Y, Yu B. Treatment seeking behavior and associated factors of suspected dengue fever among Shan people in eastern Shan special region IV, Myanmar: a cross-sectional study. BMC Health Services Research. 2020;20(1):318. https://doi.org/10.1186/s12913-020-05163-z.

15. Barrera R, Amador M, MacKay AJ. Population dynamics of Aedes aegypt and dengue as influenced by weather and human behavior in San Juan, Puerto Rico. PLoS Negl Trop Dis. 2011;5(12):e1378. https://doi.org/10.1371/ journal.pntd.0001378.

16. Rosenstock IM, Strecher VJ, Becker MH. Social learning theory and the health belief model. Health Educ Q. 1988;15(2):175-83. https://doi.org/10.11 77/109019818801500203

17. Janz NK, Champion VL, Strecher VJ. The health belief model. In: Glanz K, Rimer BK, FM L, editors. Health behavior and health education: theory, research, and practice. 3rd ed. San Francisco: Jossey-Bass; 2002.

18. Wong LP, Atefi N, AbuBakar S. Nationwide study of factors associated with public's willingness to use home self-test kit for dengue fever in Malaysia. BMC Public Health. 2016;16(1):780. https://doi.org/10.1186/s12889-016-3409-y.

19. Israel GD. Determining sample size, program evaluation and organization development. Gainesville: institute of food and agricultural sciences, University of Florida; 1992. https://florida4h.ifas.ufl.edu/. Accessed 25 May 2018.

20. Wong LP, Abubakar S. Health beliefs and practices related to dengue fever: a focus group study. PLoS Negl Trop Dis. 2013;7(7):e2310. https://doi.org/1 0.1371/journal.pntd.0002310.

21. Xu J-W, Liu H, Yaw B, Nbwi HS. The health beliefs, dengue knowledge and control behaviors among internally displaced persons versus local residents in Kachin special region II, Myanmar. PLoS Negl Trop Dis. 2020;6(6): e0008321. https://doi.org/10.1371/journal.pntd.0008321.

22. Lindsay S, Wilson A, Golding N, et al. Improving the built environment in urban areas to control Aedes aegypti-borne diseases. Bull World Health Organ. 2017;95(8):607-8. https://doi.org/10.2471/BLT.16.189688.

23. Fritzell C, Raude J, Adde A, Dusfour I, Quenel P, Flamand C. Knowledge, attitude and practices of vector-borne disease prevention during the emergence of a new arbovirus: implications for the control of chikungunya virus in French Guiana. PLoS Negl Trop Dis. 2016;10(11):e0005081. https:// doi.org/10.1371/journal.pntd.0005081.

24. Elsinga J, Lizarazo EF, Vincenti MF, Schmidt M, Velasco-Salas ZI, Arias L, et al. Health seeking behaviour and treatment intentions of dengue and fever: a household survey of children and adults in Venezuela. PLoS Negl Trop Dis. 2015;9(12):e0004237. https://doi.org/10.1371/journal.pntd.0004237.

25. Harapan H, Michie A, Mudatsir M, Sasmono RT, Imrie A. Epidemiology of dengue hemorrhagic fever in Indonesia: analysis of five decades data from the National Disease Surveillance. BMC Res Notes. 2019;12(1):350. https://doi. org/10.1186/s13104-019-4379-9.

26. Xu JW, Li Y, Yang HL, Zhang J, Zhang ZX, Yang YM, et al. Malaria control along China-Myanmar border during 2007-2013: an integrated impact evaluation. Infect Dis Poverty. 2016;5(1):75. https://doi.org/10.1186/s40249-01 6-0171-4

27. Liu H, Xu JW, Xia M, Yang MD, Li JX, Yin YJ, et al. Comparatively qualitative study of responses to dengue fever foci in communities of three ethnical minorities crossing China-Myanmar -Thailand borders. China Trop Med. 2019;19(9):1034-8 (in Chinese)

28. WHO. Global strategy for dengue prevention and control 2012-2020. Geneva: World Health Organization; 2012. accessed 27 Feb 2018

29. Bhatt S, Gething PW, Brady OJ, Messina JP, Farlow AW, Moyes CL, et al. The global distribution and burden of dengue. Nature. 2013;496(7446):504-7. https://doi.org/10.1038/nature12060.

\section{Publisher's Note}

Springer Nature remains neutral with regard to jurisdictional claims in published maps and institutional affiliations.
Ready to submit your research? Choose BMC and benefit from:

- fast, convenient online submission

- thorough peer review by experienced researchers in your field

- rapid publication on acceptance

- support for research data, including large and complex data types

- gold Open Access which fosters wider collaboration and increased citations

- maximum visibility for your research: over $100 \mathrm{M}$ website views per year

At $\mathrm{BMC}$, research is always in progress.

Learn more biomedcentral.com/submissions 